PROCEEDINGS OF THE

AMERICAN MATHEMATICAL SOCIETY

Volume 129, Number 12, Pages 3495-3499

S 0002-9939(01)06325-0

Article electronically published on July 10, 2001

\title{
LEIBENZON'S BACKWARD SHIFT AND COMPOSITION OPERATORS
}

\author{
EVGUENI DOUBTSOV
}

(Communicated by David R. Larson)

\begin{abstract}
We apply Leibenzon's backward shift to show that the composition operator on the unit ball of $\mathbb{C}^{n}$ always maps the weighted Hardy space $H_{1-n}^{2}$ into the Hardy class $H^{2}$.
\end{abstract}

\section{INTRODUCTION}

Let $B=B_{n}$ be the open unit ball of $\mathbb{C}^{n}$. In what follows we assume that $n \geq 2$, and we use the symbol $\mathbb{D}$ to denote the unit disc of $\mathbb{C}$. Suppose $\varphi: B \rightarrow B$ is holomorphic. Then the composition operator $C_{\varphi}$ is defined by $\left(C_{\varphi} f\right)(z)=f(\varphi(z))$, where $f: B \rightarrow \mathbb{C}$ is holomorphic, $z \in B$.

Classical results show that $C_{\varphi}$ is bounded on the Hardy spaces $H^{p}(\mathbb{D}), 0<p \leq \infty$ (Littlewood's subordination principle). The same situation holds for the Bergman spaces $A^{p}(\mathbb{D}), 0<p<\infty$.

In contrast with the one variable case, $C_{\varphi}$ may not induce a bounded operator on $H^{p}(B)$. This observation suggests two types of results. First, a characterization of all $\varphi$ such that $C_{\varphi}$ is bounded on $H^{p}(B)$ is obtained by B.D. MacCluer in [4]. Second, let $A_{q}^{p}(B), q>-1$, denote a (standard) weighted Bergman space. Using Carleson measures considerations and a slice integration technique, B.D. MacCluer and P.R. Mercer prove in [5] that $C_{\varphi}$ always maps $H^{p}\left(B_{n}\right)$ into $A_{n-2}^{p}\left(B_{n}\right)$. In fact, $A_{n-2}^{p}\left(B_{n}\right)$ is the "smallest" space with such a property. Further results in this direction show that $C_{\varphi}: A_{q}^{p} \rightarrow A_{q+n-1}^{p}\left(B_{n}\right)$ for all $q>-1$ (see [2]).

The elementary approach of the present note uses Leibenzon's backward shift operator. We fix a Hilbert target space $Y$, and we look for a largest function space $X$ such that $C_{\varphi}: X \rightarrow Y$. In particular, we show that $C_{\varphi}: H_{1-n}^{2}\left(B_{n}\right) \rightarrow H^{2}\left(B_{n}\right)$, where $H_{1-n}^{2}$ is a weighted Hardy space in the ball.

Notation. Normalized Lebesgue measure on the sphere $S=\partial B_{n}$ is $\sigma$. Respectively, $\nu$ is Lebesgue measure on $B, \nu(B)=1$. So $L^{p}(S)=L^{p}(\sigma)$ and $L^{p}(B)=L^{p}(\nu)$.

The norm in the classical Hardy space $H^{p}(B)$ is

$$
\|f\|_{H^{p}}^{p}=\sup _{0<r<1} \int_{S}|f(r \zeta)|^{p} d \sigma(\zeta)=\int_{S}\left|f^{*}(\zeta)\right|^{p} d \sigma(\zeta) .
$$

Received by the editors March 1, 1999.

2000 Mathematics Subject Classification. Primary 47B38.

Key words and phrases. Composition operator, Leibenzon's backward shift. 
Given $0<p<\infty$ and $q>-1$, the weighted Bergman space $A_{q}^{p}(B)$ consists of functions $f$ holomorphic in $B$ and such that

$$
\int_{B}|f(z)|^{p}\left(1-|z|^{2}\right)^{q} d \nu(z)<\infty .
$$

We use the standard multi-index notation: if $\alpha=\left(\alpha_{1}, \ldots, \alpha_{n}\right)$, then $|\alpha|=$ $\sum_{j=1}^{n} \alpha_{j}$ and $\alpha !=\prod_{j=1}^{n} \alpha_{j} !$

Definition. Let $q \geq 0$. A holomorphic function $f(z)=\sum_{\alpha} c_{\alpha} z^{\alpha}, z \in B$, belongs to the weighted Hardy space $H_{q}^{2}(B)$ if

$$
\|f\|_{q}^{2}=\sum_{\alpha}\left|c_{\alpha}\right|^{2}\left\|z^{\alpha}\right\|_{L^{2}(S)}^{2}\left(\begin{array}{c}
|\alpha|+q \\
q
\end{array}\right)^{-1}<\infty
$$

where $\left(\begin{array}{l}a \\ b\end{array}\right)=\frac{\Gamma(a+1)}{\Gamma(a-b+1) \Gamma(b+1)}$ is "the binomial coefficient"; $a \geq b$. Respectively

$$
\|f\|_{-q}^{2}=\sum_{\alpha}\left|c_{\alpha}\right|^{2}\left\|z^{\alpha}\right\|_{L^{2}(S)}^{2}\left(\begin{array}{c}
|\alpha|+q \\
q
\end{array}\right) .
$$

Note that $H_{0}^{2}=H^{2}$ (with equal norms) and $H_{q}^{2} \subset H_{r}^{2}$ if $q<r$.

Remark 1. A more general definition (cf. [1, Section 2.1) says that $H_{q}^{2}$ is the weighted Hardy space $H^{2}(\beta)$ with $\beta(j)=\left(\begin{array}{c}j+q \\ q\end{array}\right)^{-1 / 2}$. In particular, if $q>0$, then $H_{q}^{2}$ is the weighted Bergman space $A_{q-1}^{2}$, with an equivalent norm; $H_{-1}^{2}$ is a Dirichlet-type space (see [1] for details).

\section{THE EMBEDDing THEOREM}

The main goal of the present section is to establish the following result.

Theorem 1. Suppose that $\varphi: B_{n} \rightarrow B_{n}$ is holomorphic and $\varphi(0)=0$. Then $\left\|C_{\varphi} f\right\|_{0} \leq\|f\|_{1-n}$ for all $f \in H_{1-n}^{2}(B)$.

Recall that the original proof of Littlewood's subordination principle uses the backward shift operator on $\ell^{2}$ (see e.g. 6], Chapter 1). The sphere $S$ is not a group, so there is no canonical analogue of the backward shift. Nevertheless, given a holomorphic function $f$, define

$$
\left(L_{j} f\right)(z)=\int_{0}^{1} \frac{\partial f}{\partial z_{j}}(t z) d t, \quad 1 \leq j \leq n, z \in B .
$$

The "backward shifts" $L_{j} f$ were introduced by Leibenzon (see [3]) to solve the Gleason problem

$$
f(z)-f(0)=\sum_{j=1}^{n} z_{j}\left(L_{j} f\right)(z) .
$$

As we show below, the shifts $L_{j}$ are still useful in the study of composition operators. 
Proof. Assume, without loss of generality, that $f$ is a polynomial. Substitute $z$ by $\varphi(z)$ in (2.1); then we have

$$
C_{\varphi} f(z)=f(0)+\sum_{j=1}^{n} \varphi_{j}(z)\left(C_{\varphi} L_{j} f\right)(z)
$$

Since $\varphi(0)=0$, the terms on the right side are orthogonal in $H_{0}^{2}$. Hence

$$
\begin{aligned}
\left\|C_{\varphi} f\right\|_{0}^{2} & =|f(0)|^{2}+\left\|\sum_{j=1}^{n} \varphi_{j} \cdot C_{\varphi} L_{j} f\right\|_{0}^{2} \\
& \leq|f(0)|^{2}+\int_{S}\left(\sum_{j=1}^{n}\left|\varphi_{j}\right|^{2}\right)\left(\sum_{j=1}^{n}\left|C_{\varphi} L_{j} f\right|^{2}\right) d \sigma \\
& \leq|f(0)|^{2}+\sum_{j=1}^{n}\left\|C_{\varphi} L_{j} f\right\|_{0}^{2} .
\end{aligned}
$$

By induction, we obtain

$$
\left\|C_{\varphi} f\right\|_{0}^{2} \leq \sum_{k=0}^{\operatorname{deg} f} \sum_{j_{1}, \ldots, j_{k}}\left|\left(L_{j_{1}} \ldots L_{j_{k}} f\right)(0)\right|^{2} .
$$

Let $f(z)=\sum_{\alpha} c_{\alpha} z^{\alpha}$. Then the above double sum is equal to

$$
\sum_{\alpha}\left|c_{\alpha}\right|^{2} \sum_{j_{1}, \ldots, j_{|\alpha|}}\left|\left(L_{j_{1}} \ldots L_{j_{|\alpha|}} z^{\alpha}\right)(0)\right|^{2}
$$

Now, fix a multi-index $\alpha$. Consider a sequence $J=\left\{j_{1}, \ldots, j_{|\alpha|}\right\}$ and the corresponding operator $L_{J}=L_{j_{1}} \ldots L_{j_{|\alpha|}}$. Observe that $\left(L_{J} z^{\alpha}\right)(0) \neq 0$ if and only if $\#\left\{k: j_{k}=m\right\}=\alpha_{m}$ for all $1 \leq m \leq n$. A simple combinatorial calculation shows that there are $|\alpha| ! / \alpha$ ! different sequences $L_{J}$ such that $\left(L_{J} z^{\alpha}\right)(0) \neq 0$. Moreover, the value $\left(L_{J} z^{\alpha}\right)(0)$ is the same for all such $J$. Indeed, one has

$$
\left(L_{j} z^{\alpha}\right)(\zeta)=\frac{\alpha_{j}}{|\alpha|} \zeta^{\alpha-e_{j}}, \quad \text { where }\left(e_{j}\right)_{k}=\delta_{j k}, 1 \leq k \leq n .
$$

Therefore $\left|\left(L_{J} z^{\alpha}\right)(0)\right|=\alpha ! /|\alpha|$ !, and

$$
\sum_{J}\left|\left(L_{J} z^{\alpha}\right)(0)\right|^{2}=\alpha ! /|\alpha| !
$$

Recall that

$$
\left\|z^{\alpha}\right\|_{L^{2}\left(S_{n}\right)}^{2}=\frac{(n-1) ! \alpha !}{(n-1+|\alpha|) !}
$$

So, finally we obtain

$$
\left\|C_{\varphi} f\right\|_{0}^{2} \leq \sum_{\alpha}\left|c_{\alpha}\right|^{2} \frac{\alpha !}{|\alpha| !}=\sum_{\alpha}\left|c_{\alpha}\right|^{2}\left\|z^{\alpha}\right\|_{L^{2}(S)}^{2}\left(\begin{array}{c}
|\alpha|+n-1 \\
|\alpha|
\end{array}\right)=\|f\|_{1-n}^{2} .
$$

Corollary 2. For all $\varphi: B_{n} \rightarrow B_{n}$, the composition operator $C_{\varphi}$ maps $H_{1-n}^{2}\left(B_{n}\right)$ into $H^{2}\left(B_{n}\right)$.

Proof. Let $\psi$ be a holomorphic automorphism of $B_{n}$. It is well known that $C_{\psi}$ : $H^{2} \rightarrow H^{2}$. On the other hand, the automorphisms of the ball act transitively. 
Remark 2. Observe that Theorem 1 is optimal in the scale of weighted Hardy (or weighted Dirichlet) spaces. Namely, if $q>1-n$, then there exists $\varphi, \varphi(0)=0$, such that $C_{\varphi}$ does not map $H_{q}^{2}\left(B_{n}\right)$ into $H^{2}\left(B_{n}\right)$.

For example, assume $n=2$ and $q>1-n=-1$. Let $I: B_{2} \rightarrow \mathbb{D}, I(0)=0$, be an inner function, that is, $\left|I^{*}\right|=1 \sigma$-a.e. Define $\varphi(z)=(I(z), 0)$ and consider $f_{k}(z)=\sqrt{k^{1+q}} z_{1}^{k}, k \in \mathbb{N}$. Then $\left\|f_{k}\right\|_{q}^{2} \asymp 1$ for all $k$. On the other hand, we have $\left\|f_{k}(\varphi(z))\right\|_{0}^{2}=k^{1+q}\left\|I^{k}\right\|_{0}^{2}=k^{1+q}$. In other words, $C_{\varphi}$ does not map $H_{q}^{2}$ into $H^{2}$.

Proposition 3. Suppose that $\varphi: B_{n} \rightarrow B_{n}$ is holomorphic, $\varphi(0)=0$, and $q \geq 0$. Then $C_{\varphi}: H_{q+1-n}^{2}\left(B_{n}\right) \rightarrow H_{q}^{2}\left(B_{n}\right)$.

Proof. If $q=0$, then we have Theorem 1 . To avoid ugly calculations, consider only an illustrative case $q=1$. In other words, let the target space be $H_{1}^{2}=A^{2}$. Recall that

$$
C_{\varphi} f(z)=f(0)+\sum_{j=1}^{n} \varphi_{j}(z)\left(C_{\varphi} L_{j} f\right)(z)
$$

Since $\varphi(0)=0$, the terms on the right side are orthogonal in $L^{2}\left(B,|z|^{2 k} d \nu(z)\right)$ for all $k \in \mathbb{Z}_{+}$. On the other hand, by the Schwarz lemma in the ball,

$$
\sum_{j=1}^{n}\left|\varphi_{j}(z)\right|^{2} \leq|z|^{2}
$$

Therefore, by induction,

$$
\begin{aligned}
\left\|C_{\varphi} f\right\|_{1}^{2} & \leq \sum_{k \geq 0} \sum_{j_{1}, \ldots, j_{k}}\left\||z|^{2 k}\right\|_{L^{2}(B)}\left|\left(L_{j_{1}} \ldots L_{j_{k}} f\right)(0)\right|^{2} \\
& =\sum_{\alpha}\left|c_{\alpha}\right|^{2} \frac{\alpha !}{|\alpha| !} \frac{n}{|\alpha|+n} \leq 2\|f\|_{2-n}^{2} .
\end{aligned}
$$

Corollary 4. Let $\varphi: B_{n} \rightarrow B_{n}$ and $q \geq 0$. Then $C_{\varphi}: H_{q+1-n}^{2} \rightarrow H_{q}^{2}$.

Proof. Let $\psi$ be an automorphism of $B_{n}$. It is well known that $C_{\psi}: H_{q}^{2} \rightarrow H_{q}^{2}$ for all $q \geq 0$.

The appropriate choice of $q$ (cf. Remark 1) yields

Corollary 5. Let $\varphi: B_{n} \rightarrow B_{n}$. Then $C_{\varphi}: H^{2} \rightarrow A_{n-2}^{2}$ and $C_{\varphi}: A_{r}^{2} \rightarrow A_{r+n-1}^{2}$ for all $r>-1$.

\section{REFERENCES}

1. C.C. Cowen and B.D. MacCluer, Composition operators on spaces of analytic functions, CRC Press, Boca Raton, 1995. MR 97i:47056

2. J.A. Cima and P.R. Mercer, Composition operators between Bergman spaces on convex domains in $\mathbb{C}^{n}$, J. Operator Theory 33 (1995), 363-369. MR 96h:47036

3. G.M. Henkin, Approximation of functions on pseudoconvex domains and Leibenzon's theorem, Bull. Acad. Polon. Sci. Sér. Sci. Math. Astronom. Phys. 19 (1971), 37-42. MR 44:4234

4. B.D. MacCluer, Compact composition operators on $H^{p}\left(B_{N}\right)$, Michigan Math. J. 32 (1985), 237-248. MR 86g:47037 
5. B.D. MacCluer and P.R. Mercer, Composition operators between Hardy and weighted Bergman spaces on convex domains in $\mathbb{C}^{n}$, Proc. Amer. Math. Soc. 123 (1995), 2093-2102. MR 95i: 47060

6. J.H. Shapiro, Composition operators and classical function theory, Springer-Verlag, New York, 1993. MR 94k:47049

Department of Mathematics, Michigan State University, East Lansing, Michigan 48824

Current address: ul. Partizana Germana 14/117, kv. 335, 198205 St. Petersburg, Russia

E-mail address: ed@ED8307.spb.edu 